\title{
Which test, coronary angiography or colonoscopy?
}

\author{
Ilaria Cecioni · Filippo Fassio - Chiara Alamanni · \\ Fabio Almerigogna $\cdot$ Pietro Amedeo Modesti
}

Received: 18 November 2008/ Accepted: 20 November 2008/Published online: 12 December 2008

(C) SIMI 2008

\section{Case report}

\section{Ilaria Cecioni, Filippo Fassio, Chiara Alamanni, Fabio Almerigogna}

A 87-year-old man was conducted to the Emergency Unit after a syncope. At admittance, the patient was awake, asymptomatic for chest pain, Troponin I values were $0.02 \mu \mathrm{g} / \mathrm{L}$, Glasgow coma scale (GCS) score was 15, the blood pressure 110/ $55 \mathrm{mmHg}$, the pulse 90 beats per minute, the peripheral oxygen saturation $98 \%$. Laboratory tests only showed microcytic hypochromic anemia [hemoglobin level $9.8 \mathrm{~g} / \mathrm{dL}$; hematocrit $31.1 \%$; mean cell volume (MCV) $77.7 \mathrm{fL}$; mean cell hemoglobin (MCH) $24.3 \mathrm{pg}$; mean concentration of hemoglobin per volume of red cells (MCHC) $31.3 \mathrm{~g} / \mathrm{dL}]$. He reported assumption of sublingual nitrates some minutes before the syncope for chest pain caused by a mild effort (Valsalva).

Six hours after admittance, the patient was still asymptomatic, his conditions appeared stable, Troponin I values remained within the normal range and he was transferred to our medical ward to complete the diagnostic and therapeutic work-up.

Relevant past history included, 11 years before, demonstration of coronary artery disease (CAD) [75\% stenosis of

I. Cecioni · F. Fassio - C. Alamanni · F. Almerigogna Division of Immunology and Cellular Therapies,

Department of Internal Medicine,

University of Florence, Florence, Italy

P. A. Modesti ( $\square)$

Department of Critical Care Medicine and Surgery, University of Florence and Don Carlo Gnocchi Foundation, IRCCS, Viale Morgagni 85,

50134 Florence, Italy

e-mail: pamodesti@unifi.it left circumflex and first obtuse marginal arteries, 50\% stenosis of mid left anterior descending artery, and subcritical stenosis $(<50 \%)$ of second obtuse marginal artery], treated with medical therapy. Few months after diagnosis of CAD, acetylsalicylic acid (ASA) was substituted by ticlopidine because of melena and heartburn; at that time an esophagogastroduodenoscopy (EGDS) showed no lesions. Thereafter the patient suffered from chronic angina with stable threshold (functional classification class II of the Canadian Cardiovascular Society) relieved by nitrates. Five years before the admission a microcytic hypochromic anemia was demonstrated, an EGDS was repeated and showed chronic atrophic gastritis with Helicobacter-like organism (HLO) +, eradication therapy was started but discontinued for adverse effects. A contemporary gradual reduction of the anginal threshold was noted. Six months before the admission anorexia, with weight loss of about $7 \mathrm{~kg}$, and constipation developed.

Physical examination revealed: pale discoloration of skin, high-frequency low-grade systolic murmur audible on all cardiac auscultation areas, a non-tender non-pulsatile roundish mass deeply palpable in the right iliac fossa of several (6-8) centimetres of diameter, of increased consistency and preserved mobility on abdominal wall. Ultrasound examination of the abdomen was performed and revealed a kidney-shaped mass of $7 \times 7 \times 8 \mathrm{~cm}$ dimensions, suggesting a right colon cancer.

Colonoscopy showed an ulcerated cauliflower-shaped lesion in the cecum occupying the whole intestinal lumen. The histological examination of a biopsy specimen confirmed the diagnosis of the adenocarcinoma of the colon.

CT scan of the abdomen showed a large irregular thickening of the cecum wall with regional lymph nodes involvement without signs of distant metastases. 


\section{Comment}

\section{Pietro Amedeo Modesti}

Throughout the history and development of medical practice, the physical examination of the abdomen occupied a pivotal role in both diagnostic and treatment paradigms. In more recent times, however, its value in the clinic has dwindled. Technical skill is known and is retained by successful medical students in different countries. The technique of physical examination is essential to pass MRCP (Membership of the Royal Colleges of Physicians of the United Kingdom) [1] and FRCS (Fellowship of the Royal College of Surgeons) [2] examinations. Getting the right answer by the wrong technique will result in failure but the wrong answer by the correct technique can still produce a pass.

Knowledge of the full routine is important although in common clinical practice it is not required in full every time. A well-conducted abdomen examination may indeed reveal a great deal of information. Conversely, a poorly performed examination can reveal nothing and lose the patient's confidence, if it causes pain. Everyone develops a personal technique with time and adjusts it according to circumstances. However, today many practitioners rarely use it in practice. A different importance is indeed perceived if the problem is an acute abdomen or routine because abdominal examination is rarely performed in patients without abdominal pain. It is often not considered that abdominal masses are only occasionally presented by patient usually being detected at physical examination.

When performing abdominal examination, supraclavicular and inguinal nodes should be searched. Likewise scars (especially round umbilicus for laparoscopy scars), distension, prominent veins, local swelling, pulsation, visible peristalsis, skin lesions, asymmetrical movement at eye level should be considered at inspection and lesions of abdominal wall excluded. When palpation is performed (warm hands) the tender areas should be examined last. Light palpation then deep. Check for guarding, rigidity and rebound tenderness. Finally, for any mass should be determined site, tenderness, size and shape, surface (irregular or smooth), edge (regular or irregular), consistency (soft or hard), mobility, whether pulsatile or ballotable. Limitations may exist, obesity making the exercise very difficult. It may seem like the fairytale princess asked to feel a pea under 20 mattresses. When an abdominal mass is discovered, its nature must be defined. Using a systematic approach often permits the identification of the mass before the use of sophisticated tests.

Where is the mass located? A practical approach is to divide the abdomen into four quadrants. Starting from the principle that an abdominal mass originates from an organ, surface anatomy may suggest which one is enlarged. A mass seen in the left lower quadrant, for example, could be of colonic or ovarian origin but, unless there is situs inversus, one would not consider an appendiceal abscess!

Does the mass move with respiration? In the upper abdomen, a mobile intra-abdominal mass will move downward with inspiration, while a more fixed organ (e.g., aorta, pancreas) or an abdominal wall mass (e.g., hematoma of rectus muscle) will not. The contour and surface of the mass is achieved by inspection, percussion and palpation. Is the organ air-filled (e.g., stomach) or fluid-filled? Is it a well-defined mass (e.g., liver, spleen) or are its borders difficult to define (matted loops of small bowel)? Is the surface regular? An enlarged liver due to fatty infiltration may have a smooth surface, while a cirrhotic organ is usually irregular and nodular. What is the consistency of the mass? Firm? Hard or soft? Is it pulsatile? In the absence of ascites, ballottement of an organ situated in either upper quadrant more likely identifies an enlarged kidney (more posterior structure) than hepatomegaly or splenomegaly.

A mass in the right lower quadrant has its origin either in a pelvic structure (ovary, uterus, fallopian tube) or in the lower GI tract (colon, distal small bowel, appendix) (Table 1). Masses originating from pelvic organs commonly are detected in woman and bimanual palpation is the preferred method. Conversely, the deeper organs in the lower GI tract are usually ill-defined. Clinical context, history and general physical examination may be of special help. Although an abdominal mass may be discovered during physical examination of an asymptomatic individual, in a patient presenting with anemia and recent history of weight loss, a right lower quadrant mass would suggest bowel disease. Inflammatory bowel disease usually would be associated with pain on palpation but carcinoma of the cecum would be painless and is usually associated with occult blood loss and anemia.

Physical examination modified the perspective immediately, raising the question whether coronary angiography

Table 1 Abdominal masses detected in the right iliac fossa

Actinomycosis

Amebic abscess

Appendix mass or abscess

Cecal cancer or distension

Crohn's disease

Hernia

Ileocecal tuberculosis mass

Intussusception

Kidney abnormality

Ovarian tumour

Tumour in intra-abdominal testicle 
is to be performed or not before surgery. Specific guidelines [3] exist for our patient which can be followed at this point. The sailor must fix the position before deciding the ship's bearing.

Conflict of interest statement The authors declare that they have no conflict of interest related to the publication of this manuscript.

\section{References}

1. MRCP(UK) EXAMINATION WEBSITE. http://www.mrcpuk.org/ Pages/Home.aspx. Accessed on 17 November 2008
2. The Royal College of Surgeons of England. Examinations. http://www.rcseng.ac.uk/exams. Accessed 17 November 2008

3. Fleisher LA, Beckman JA, Brown KA et al (2007) ACC/AHA 2007 Guidelines on perioperative cardiovascular evaluation and care for noncardiac surgery: executive summary: a report of the American College of Cardiology/American Heart Association Task Force on Practice Guidelines (Writing Committee to revise the 2002 guidelines on perioperative cardiovascular evaluation for noncardiac surgery): Developed in collaboration with the American Society of Echocardiography, American Society of Nuclear Cardiology, Heart Rhythm Society, Society of Cardiovascular Anesthesiologists, Society for Cardiovascular Angiography and Interventions, Society for Vascular Medicine and Biology, and Society for Vascular Surgery. Circulation 116:1971-1996 